\title{
Three-Dimensional Polarization by Means of Scanning HOLZ-CBED Technique.
}

\author{
Roberto dos Reis ${ }^{1}, \mathrm{~S}-\mathrm{L} \mathrm{Hsu}^{1,2}$, C. Ophus ${ }^{1}$, R. Ramesh ${ }^{2,3}$ and J. Ciston ${ }^{1}$
}

1. National Center for Electron Microscopy, Molecular Foundry, Lawrence Berkeley National Laboratory, Berkeley, CA, USA

2. Department of Materials Science \& Engineering, University of California, Berkeley, CA, USA

3. Materials Sciences Division, Lawrence Berkeley National Laboratory, Berkeley, CA, USA

4. Energy Technologies, Lawrence Berkeley National Laboratory, Berkeley, CA, USA

Convergent-beam electron diffraction (CBED) patterns can contain diffracted beams from higher-order Laue zones (HOLZ) in addition to the more often observed diffracted beams from the zeroth-order Laue zone (ZOLZ) that includes the origin of the reciprocal lattice. However, structural information based only on the ZOLZ is insensitive to the strain-field components in the direction of the electron beam. HOLZ diffraction vectors possess components along the incident electron beam direction and are sensitive to components of static real lattice displacement fields that lie along the incident electron beam direction [1]. In this work, we will discuss the usefulness of HOLZ-CBED in combination with recently developed scanning diffraction techniques using fast-pixelated electron detectors in order to map strain and polarization fluctuation along the beam direction.

As an example, we have used the information contained in the HOLZ diffraction to investigate the polarization vector in $\mathrm{ABO}_{3}$ perovskite oxide superlattices [2-4]. Such information is essential to understand the underlying physics of the polar vortices formation in the recently discovered phenomena that emerge from interfacial or confinement effects in artificial ferroelectric $(\mathrm{SrTiO} 3)_{\mathrm{n}} /(\mathrm{PbTiO} 3)_{\mathrm{n}} /(\mathrm{SrTiO} 3)_{\mathrm{n}}$ symmetric tri-layer $(\mathrm{STO} / \mathrm{PTO} / \mathrm{STO})$ superlattices [5]. The continuous rotation of vortices exhibits a polarization $(\mathbf{P})$ in-plane $\mathrm{p}_{1} \mathbf{x}$ and $\mathrm{p}_{2} \mathbf{y}$ and out-of-plane $\mathrm{p}_{3} \mathbf{Z}$ components.

Figure 1 shows the effect of purely in- and out-of plane polarization, respectively, $\mathbf{P}=\mathbf{x}$ and $\mathbf{P}=\mathbf{z}$, in simulated HOLZ patterns for a trilayer $(\mathrm{STO})_{n} /(\mathrm{PTO})_{n} /(\mathrm{STO})_{n}$ with $\mathrm{n}=20$ unit cells (see model in the first row) observed along the [001] pseudocubic (pc) zone axis. First and second order Laue zones (FOLZ and SOLZ) are present in both cases depicting the out-of-plane components. The effect of outof-plane polarization is strongly observed in the splitting of HOLZ ring (indicated by the white arrow in (b)). This effect is originated by the increase of the periodicity along the beam direction (001)pc as the symmetry breaks due to the relative shift of the $\mathrm{A}$ and $\mathrm{B}$ sites ( $\mathrm{Pb}$ and $\mathrm{Ti}$, in this case). In contrast, the FOLZ diffraction intensity asymmetry increases for in-plane polarization case. These features would allow us to separate both type of polarizations within HOLZ diffraction.

The feasibility of the method was tested by acquiring experimental data as 4D-STEM datasets [6] from STO/PTO/STO symmetric tri-layer grown by pulsed laser deposition on $\mathrm{DyScO} 3$ (001)pc substrate to probe polarization of confined PTO layer. CBED patterns were recorded at many probe positions with millisecond dwell times by using a Gatan K2-IS direct electron detection camera installed on the TEAM I microscope [3] with sample kept at $\mathrm{T}=70 \mathrm{~K}$. The microscope voltage was set to $300 \mathrm{kV}$ and the STEM probe convergence angle to 1.6 millirads. The high-angle annular dark field (HAADF) signal was recorded simultaneously using a monolithic Fischione detector. All data processing was performed using custom codes written in MATLAB. 
Figure 2 presents experimental HOLZ-CBED diffraction patterns averaged from 20200 data points over a region of 45 by $90 \mathrm{~nm}$ comprising different domains regions. A clusterization procedure was applied to enhance the signal-to-noise of the HOLZ diffraction. Presence of polarization along the beam direction can be observed in the ring split and intensity reduction of FOLZ ring (pointed by the arrows). Application of modified multislice and bloch wave simulations in order to precisely reproduce the effects in the HOLZ diffraction will also be discussed [8].

\section{References:}

[1] R Carpenter and J Spence, Acta Crystallographica Section A: Crystal Physics, Diffraction, Theoretical and General Crystallography 38 (1982), p. 55.

[2] P. Zubko et al, Ann. Rev. Cond. Matter Phys. 2 (2011), p. 141-165.

[3] M. Dawber, K.M. Rabe, J.F. Scott, Rev Mod Phys 77 (2005), p.1083-1130.

[4] J. Mannhart, D.G. Schlom, Science 327 (2010), p.1607-1611.

[5] A.K. Yadav, et al., Nature 530 (2016), p. 198-20.

[6] C. Ophus, et al. Acta Cryst A70 (2014), p. C1455.

[7] EJ Kirkland, Advanced Computing in Electron Microscopy 2nd Edition (2010) Springer.

[8] The work at the Molecular Foundry was supported by the Office of Science, Office of Basic Energy Sciences, of the U.S. Department of Energy under Contract No. DE-AC02-05CH11231. R.dR. and J.C. acknowledge support from the U.S. Department of Energy Early Career Research Program.
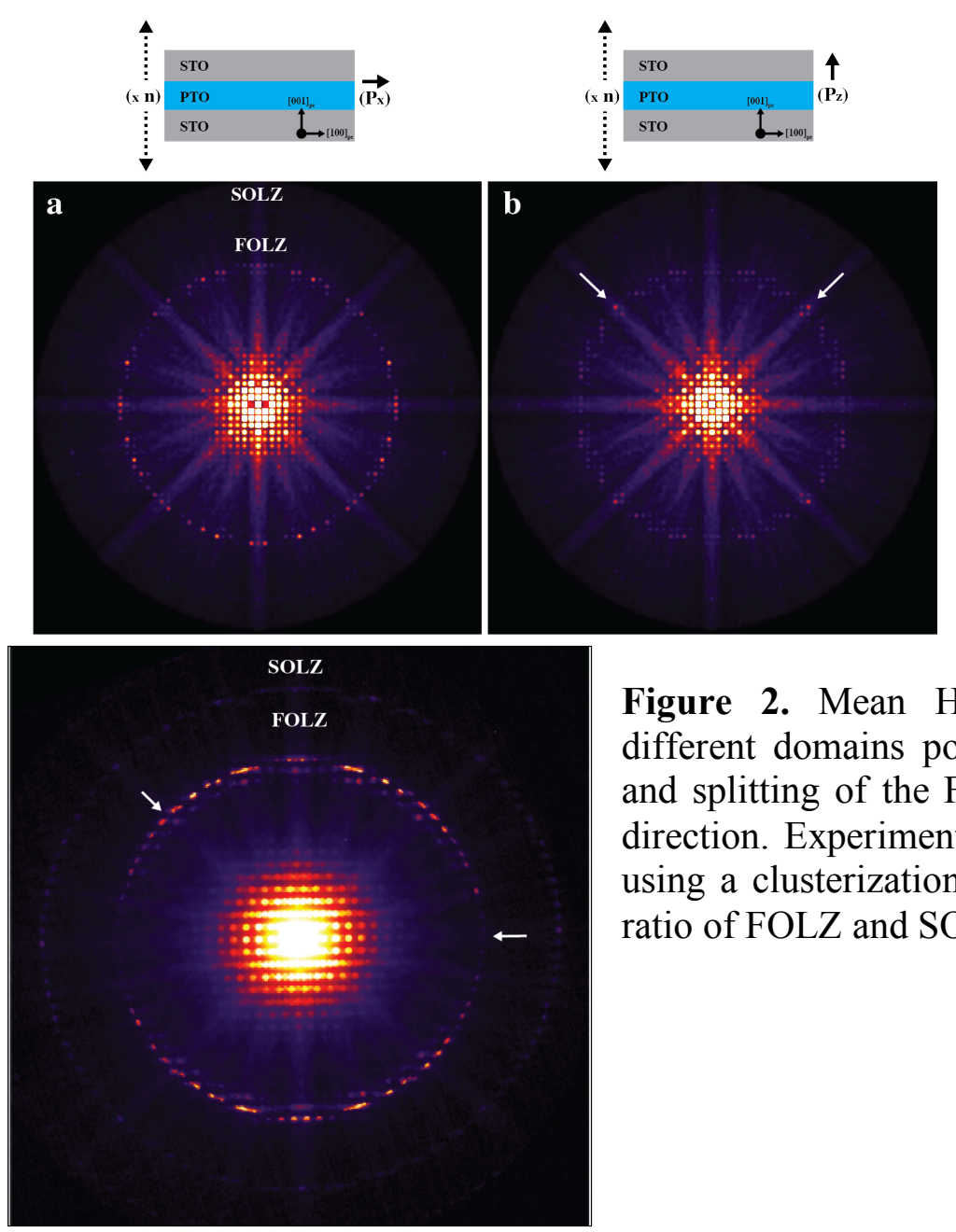

Figure 1. HOLZ-CBED simulation for (a) in-plane $\mathrm{P}=\mathrm{x}$ and (b) out-of-plane $\mathrm{P}=\mathrm{z}$, polarizations following the schematic draws in the top row. A modified multislice method was applied taking into account the term containing the second derivative with respect to $\mathrm{z}$ in the Schröedinger equation for the full wave function $\psi$ as a function of three spatial coordinates $(x, y, z)$ in an electrostatic potential $\mathrm{V}(\mathrm{x}, \mathrm{y}, \mathrm{z})$ of the specimen [7]. FOLZ and SOLZ lines are depicted in (a), and the split of FOLZ line in the out-of-plane case is pointed by the arrows.

Figure 2. Mean HOLZ-CBED pattern depicting overlap of different domains pointed by the arrows. The reduced inyensity and splitting of the FOLZ ring indicates polarization along beam direction. Experimental HOLZ diffraction patterns were denoised using a clusterization procedure that enhances the signal-to-noise ratio of FOLZ and SOLZ diffraction. 